\title{
Factors linked to glenohumeral bone and cartilage lesions during anterior shoulder stabilization surgery
}

Kyle R. Duchman

Carolyn M. Hettrich

Natalie A. Glass

Robert W. Westermann

Brian R. Wolf

Keith Baumgarten

Julie Bishop

Jonathan Bravman

Robert Brophy

James Carpenter

Grant Jones

John Kuhn

C. Benjamin Ma

Robert Marx

Eric McCarty

Bruce Miller

Matthew Smith

MD

McCarty Eric

MD

Bruce Miller

MD

MS

Matthew Smith

Rick Wright

Alan Zhang

\section{Video Abstract}

Keywords: shoulder instability, shoulder dislocation, glenoid bone loss, glenohumeral joint, Bankart, anterior instability, shoulder stabilization, Hill-Sachs, lesion, humeral head, labrum, chondrosis, defects, cartilage, bone, primary surgery, revision surgery, Multicenter Orthopaedic Outcomes Network, The American Journal of Sports Medicine, University of lowa 
Posted Date: September 20th, 2019

DOl: https://doi.org/10.21203/rs.2.15077/v1

License: (c) (i) This work is licensed under a Creative Commons Attribution 4.0 International License. Read Full License 


\section{Abstract}

Anterior shoulder instability is frequently linked to changes in the glenohumeral joint, including bone and cartilage lesions. But the independent factors associated with lesion occurrence aren't well known. To address this gap, researchers from the Multicenter Orthopaedic Outcomes Network, or MOON, Shoulder Group have compared the demographic characteristics, clinical outcomes, and intraoperative findings of patients undergoing primary or revision shoulder stabilization surgery. Their results should help orthopedic surgeons better anticipate the likelihood of encountering bone loss and cartilaginous defects during surgery, which could improve treatment. The team performed a cross-sectional study of data from 545 participants enrolled in the MOON Shoulder Group database. Demographic data and patient-reported outcomes were collected preoperatively using standard questionnaires, and intraoperative findings were contributed by 25 orthopedic surgeons practicing at 11 United States institutions. All patients underwent either primary or revision arthroscopic or open shoulder stabilization surgery. The researchers found that several demographic and operative variables differed between the primary and revision groups. Notably, the patients undergoing revision surgery were older, were more often smokers, described more preoperative instability events, and were more likely to require reduction assistance after an instability event. But the most striking difference between the groups was the high frequency of bone and cartilage lesions in patients undergoing revision surgery. The majority of these lesions were characterized by glenoid bone loss involving over $10 \%$ of the glenoid width. The team used multivariate logistic regression analysis to identify the factors independently associated with the occurrence of these lesions at the time of surgery. The results revealed that revision surgery, increasing age, male sex, Short-Form 36 Physical Component Score, black race, and increasing body mass index were all tied to the presence of bone and cartilage lesions at the time of surgery. Of these, revision surgery, male sex, and black race showed the strongest associations. These findings suggest that a thoughtful preoperative workup, including advanced diagnostic imaging, should be performed on patients undergoing shoulder stabilization surgery. Pinpointing the factors that may lead to the presence of glenohumeral bone and cartilage lesions may help orthopedic surgeons better counsel patients and potentially optimize clinical outcomes. 